\title{
Stability Test of Networked Systems with Multiple Delays
}

\author{
Yang Xiao, Jinfeng Kou \\ Institute of Information Science \\ Beijing Jiaotong University \\ Beijing 100044, China \\ Emails: yxiao@bjtu.edu.cn, xiaokouzi922@126.com
}

Received: April 20, 2019. Revised: May 30, 2021. Accepted: October 29, 2021. Published: November 17, 2021.

\begin{abstract}
In this paper, we propose a sufficient stability condition for networked systems with multiple delays based on the 2-D polynomials and 2-D Hurwitz-Schur stability. The main advantage of the new stability condition is that it is applicable to the general case of networked systems with multiple, incommensurate delays yet numerically tractable. The characteristic polynomials of networked systems are mapping into 2-D hybrid polynomials, then to test the Hurwitz-Schur stability can. determine the networked systems, examples including system simulations verify the validity of the proposed test algorithms.
\end{abstract}

Index Terms-Networked systems, quasipolynomials, 2-D hybrid polynomials, 2-D Hurwitz-Schur stability test

\section{INTRODUCTION}

In many engineering applications, including networked systems, time delay is a so urce of instability [1-22]. In the networked systems, the individual subsystems are communicating with each other via digital networks, like Internet or wireless LAN. Obviously, these networks induce some communication delay that typically worsens the overall system performance, thus, networked systems can be regarded as a class of tim e-delay systems. However, this important time-delay property is often neglected, e.g. [21], or it is taken into account under conservative assumptions, e.g. modeling constant delays that are id entical for a 11 communication channels, e.g. [22]. Therefore, the networked systems with severe time-delay may face gr eat challenges in achie ving desired stability. In our work, we assume that all communication delays are uncertain but of upper-bound. This can be justified by a sui table communication protocol. Moreover, the delays may be different for all channels but have upper limits. This motivates our interest in studying the stability of networked systems with multiple delays. Unfortunately, the stability analysis of networked systems with multiple delays is a difficult and very tricky problem.

There exist some stability analysis tools based on Lyapunov-Krasovskii theorems and linear matrix inequality (LMI) techniques[8-15], however, proper selection of Lyapunov-Krasovskii functional is crucial for deriving stability conditions [16]. Different Lyapunov-Krasovskii functions may result in different stability conditions with different conservatism and advantage. The second problem of LMI techniques lies on the huge complexity of constructing the LMI equations for multiple time-delay systems.

Different from the LMI techniques based on LyapunovKrasovskii theorems, 2-D Hurwitz-Schur stability test of 2-D hybrid polynomial $B(s, z)$ provides a n ew approach to determine the stability of linear time-delay systems [1-3,1719], and the two problems of LMI techniques may be solved by the proposed 2-D approach in this paper. Though the results of [17-19] are derived from 2-D polynomial $B(s, z)$, the delays of the time-delay systems need to be random, and independent from zero to infinite. The results [17-19] of delay systems are not suitable for the net worked systems whose delays are finite and of upper bound.

Similar to the 2-D approaches for time-delay systems [1-3], this paper transforms the characteristic polynomial of the networked systems into 2-D polynomial $B(s, z)$, then develops a st ability test th eorem based 2-D polynomial $B(s, z)$, which is a sufficient condition for the stability of the networked systems with multiple delays. Examples including system simulations are provided to verify the validity of the proposed theorems.

\section{PROBLEM STATEMENT}

The networked systems with multiple delays can be defined by state-space model, the state-space model is described as

$$
\begin{aligned}
& \dot{\mathbf{x}}(t)=\mathbf{A}_{0} \mathbf{x}(t)+\sum_{k=1}^{K} \mathbf{A}_{k} \mathbf{x}\left(t-\tau_{k}\right)+\sum_{k=1}^{K} \mathbf{B}_{k} \mathbf{u}\left(t-\tau_{k}\right) \\
& \mathbf{y}(t)=\mathbf{C x}(t)+\mathbf{D u}(t)
\end{aligned}
$$

where $\mathbf{A}_{k}, k=0,1, \ldots, K$ and $\mathbf{B}_{m}$ are $\left(N_{A} \times N_{A}\right)$ and $\left(N_{A} \times N_{B}\right)$ constants' matrices, respectively; $\mathbf{u}(t)$ and $\mathbf{y}(t)$ are the input signal vector and output signal vector of the time-delay system, respectively; the delays $\tau_{k} \in\left[0, \bar{\tau}_{k}\right]$, $\bar{\tau}_{k}$ is a finite constant, $k=1,2, \ldots, K$. 
Taking Laplace transform to the system (1), we can get the solution of the networked system with multiple delays in $s$ domain as follows

$$
\begin{aligned}
& \mathbf{X}(s)=\left(s \mathbf{I}-\mathbf{A}_{0}-\sum_{k=1}^{K} \mathbf{A}_{k} \mathrm{e}^{-s \tau_{k}}\right)^{-1} \\
& \cdot\left(\mathbf{X}(0)+\sum_{k=1}^{K} \mathbf{B}_{k} \mathrm{e}^{-s \tau_{k}} \mathbf{U}(s)\right) \\
& \mathbf{Y}(s)=\mathbf{C}(s) \mathbf{X}(s)+\mathbf{D U}(s)
\end{aligned}
$$

The characteristic polynomial of the networked systems in (1) can be obtained from (2),

$$
B(s)=\operatorname{det}\left(s \mathbf{I}-\mathbf{A}_{0}-\sum_{k=1}^{K} \mathbf{A}_{k} \mathrm{e}^{-s \tau_{k}}\right)
$$

The characteristic polynomial (2) can be extended into

$$
B(s)=\sum_{m=0}^{N_{A}} b_{m, 0} s^{m}+\sum_{k=1}^{K} \sum_{m=0}^{N_{A}} b_{m, k} s^{m} e^{-\tau_{k} s}
$$

The characteristic polynomials of the networked system with multiple delays are quasipolynomials, and the roots of the quasipolynomials are continuous and varying in complex plane with the delay, which leads to be difficult to determine the stability of $\mathrm{n}$ etworked systems, especially for multiple delay systems. The quasi-polynomial (4) has infinite number of roots, but any bounded region of the complex plane contains only a finite number of them. Roots that are far from the origin can be assi gned to a finite number of asymptotic chains.

\section{MAIN RESULTS}

The stability of networked systems in (1) depends on the location of the roots of their characteristic polynomials (4), and it is shown as following theorem.

Theorem 1: The networked systems in (1) are asymptotically stable if and only if

$$
B(s)=\sum_{m=0}^{N_{A}} b_{m, 0} s^{m}+\sum_{k=1}^{K} \sum_{m=0}^{N_{A}} b_{m, k} s^{m} e^{-\tau_{k} s} \neq 0, \operatorname{Re} s \geq 0
$$

where $\tau_{k} \in\left[0, \bar{\tau}_{k}\right], \bar{\tau}_{k}$ is a finite constant, $k=1,2, \ldots, K$.

Proof: Compared the system (1) with the time-delay systems in [1-3, 17-19], we know that the networked systems can be regarded as a class of time-delay systems. Thus the stability theorem for time-delay systems is suitable for the system (1).

Q.E.D.

Theorem 1 established the stability relationship of networked systems and their characteristic polynomials, and it is a 1-D stability test for the quasi-polynomials in (4).

Theorem 1 is a necessary and su fficient condition for the stability of networked systems in (1), while its test is a numerical algorithm with infinite computation amount. To solve the problem, this paper tries to develop a test algo rithm based 2-D polynomial $B(s, z)$.
To the quasi-polynomials (4), we can associate the polynomials with two independent complex variables $\mathrm{s}$ and $\mathrm{z}$ given by $[1,2]$

$$
B(s, z)=\sum_{m=0}^{N_{A}} b_{m, 0} s^{m}+\sum_{k=1}^{K} \sum_{m=0}^{N_{A}} b_{m, k} s^{m} z^{-\tau_{k}}
$$

where $z=\mathrm{e}^{s} . \tau_{k} \in\left[0, \bar{\tau}_{k}\right], \bar{\tau}_{k}$ is a fi nite constant, $k=1,2, \ldots, K$.

In this paper, we de velop following theorem with the two-variable polynomial $B(s, z)$ to determine the stability conditions (5).

Theorem 2: the quasi-polynomial with multiple delays in (4) is stable if

(i) $B(1, z)=\sum_{m=0}^{N_{A}} b_{m, 0}+\sum_{k=1}^{K} \sum_{m=0}^{N_{A}} b_{m, k} z^{-\tau_{k}} \neq 0$ for $|z| \geq 1$

and

(ii) $B\left(s, \mathrm{e}^{\mathrm{j} \omega}\right)=\sum_{m=0}^{N_{A}} b_{m, 0} s^{m}+\sum_{k=1}^{K} \sum_{m=0}^{N_{A}} b_{m, k} s^{m} e^{-\mathrm{j} \omega \tau_{k}} \neq 0$

for all $\omega \in[0,2 \pi]$ and $\operatorname{Re} s \geq 0, \tau_{k} \in\left[0, \bar{\tau}_{k}\right], \bar{\tau}_{k}$ is a finite constant, $k=1,2, \ldots, K$.

The proof of Theorem 2 needs following Lemmas. Lemma 1 changes the 1-D stability test of quasi-polynomials into that of two-variable polynomial $B(s, z)$ [1-3].

Lemma 1: Given the two-variable polynomial $B(s, z)$ defined by (6), the quasi-polynomial with interval delays in (4) is stable if

$B(s, z)=\sum_{m=0}^{N_{A}} b_{m, 0} s^{m}+\sum_{k=1}^{K} \sum_{m=0}^{N_{A}} b_{m, k} s^{m} z^{-\tau_{k}} \neq 0, \operatorname{Re} s \geq 0,|z| \geq 1$

Proof: Assume that $S_{0}$ and $z_{0}$ are the roots of $B(s, z)$, according to (9), $B\left(s_{0}, z_{0}\right)=0$, we will have $\operatorname{Re} s_{0}<0,\left|z_{0}\right|<1$. It means that (9) is equivalent to $B\left(s_{0}, z_{0}\right)=0, \operatorname{Re} s_{0}<0,\left|z_{0}\right|<1$ for $\tau_{k} \in\left[0, \bar{\tau}_{k}\right], \bar{\tau}_{k}$ is a finite constant, $k=1,2, \ldots, K$.

From (6), we know that the quasi-polynomial $B(s)=\sum_{m=0}^{N_{A}} b_{m, 0} s^{m}+\sum_{k=1}^{K} \sum_{m=0}^{N_{A}} b_{m, k} s^{m} e^{-\tau_{k} s}$ is transformed into 2-D polynomial

$$
B(s, z)=\sum_{m=0}^{N_{A}} b_{m, 0} s^{m}+\sum_{k=1}^{K} \sum_{m=0}^{N_{A}(M)} b_{m, k} s^{m} z^{-\tau_{k}}
$$


by letting $Z=\mathrm{e}^{s}[1,2,7,8]$, so the quasi-polynomial $B(s)$ is a branch function of $B(s, z)$ due to $z=e^{s}$. Therefore the stability of $B(s, z)$ is a sufficient condition of that of $B(s)$, and it means that the condition (9) means the condition (5):

$$
B(s)=\sum_{m=0}^{N_{A}} b_{m, 0} s^{m}+\sum_{k=1}^{K} \sum_{m=0}^{N_{A}} b_{m, k} s^{m} e^{-\tau_{k} s} \neq 0, \operatorname{Re} s \geq 0 .
$$

Q.E.D.

The condition (9) of Lemma 1 is 2-D Hu rwitz-Schur stability of two-variable polynomial [1-3], an algebraic test algorithm for the stability of $B(s, z)$ is provided by [1-3].

Lemma 2: Given the two-variable polynomial $B(s, z)$ defined by (13) or (14), it is stable iff

(i) $B(1, z) \neq 0$ for $|z| \geq 1$

and

(ii) $B\left(s, \mathrm{e}^{\mathrm{j} \omega}\right) \neq 0$ for all $\omega \in[0,2 \pi]$ and $\operatorname{Re} s \geq 0$

The proof of Lemma 2 needs following lemma.

Lemma 3: Assume that $f(z)$ is a non-constant algebraic function such that $\operatorname{Re} f(z) \neq 0$ for $|z| \geq 1$. Then $t$ he minimum value $\operatorname{Re} f(z)$ for $|z| \geq 1$ can not occ ur at some $Z$ where $|z|>1$, where $\operatorname{Re} f$ denotes the real part absolute value of the complex function $f$.

Proof: Suppose that the minimum value of $\operatorname{Re} f(z)$ for $|z| \geq 1$ occurs at some point $z=a$, where $\operatorname{Re} f(a)>0$, and it occurs as one of the values on the branch $f_{1}$ of $f(z)$. The values of $f_{1}(z)$ near " $a$ ” on a disk of radius $r$ about “ $a$ ” (so small that it is en tirely within $|z|>1$ ) are given by $g(u)=\sum_{i=N}^{\infty} a_{i} u^{i}$, where $z=a+u^{r},|z-a| \leq \delta$.

The number $r$ is a fixed-positive integer determined only by $f_{1}$. Since by assumption $f_{1}(z)$ is bounded for $|z| \geq 1$, it follows that $N \geq 0$. Therefore, as a function of the complex variable $u$, the function $g(u)$ is bounded and analytic for $|u| \leq \delta^{\frac{1}{r}}$. Moreover, it fo llows that the values $f_{1}(z)$ for $|z-a| \leq \delta$ are given by $g(u)$ for $|u| \leq \delta^{\frac{1}{r}}$.

In fact for each value of $f_{1}(z)$ for $|z-a| \leq \delta$ there exists $u,|u| \leq \delta^{\frac{1}{r}}$ such that $|u|=|z|$ and $f_{1}(z)=g(u)$.
Since $g(u)$ is bounded and an alytic, $\operatorname{Re} g(u)$ cannot reach its $m$ inimum value for $|u| \leq \delta^{\frac{1}{r}}$ at $u=0$. Therefore, $\operatorname{Re} f_{1}(z)$ cannot have its minimum value at $z=a$, a contradiction. Therefore, it canno $\mathrm{t}$ happen that $\operatorname{Re} f(z)$ assumes its minimum value for some $Z$ where $|Z|>1$.

Q.E.D.

Proof of Lemma 2: The necessity is obvious, since (9) means that conditions (10) and (11) is satisfied [1,2].

It is su fficient to prove that con ditions (10) and (11) imply condition (9), i.e. it is suffici ent to prove that conditions (10) and (11) imply that if $|z|>1$ and $B\left(s_{1}, z\right)=0$, then $\operatorname{Re} s_{1}<0$.

Let $s=f(z)$ be the algebraic function determined by $B(s, z)=0$ in (9). Condition (10) implies that $f(z) \neq 0$ if $|z| \geq 1$; condition (11) implies that $\operatorname{Re} f(z)<0$ if $|z|=1$ and $B(s, z)=0$. Therefore, by Lemma 3 , it fo llows that $\operatorname{Re} f(z)<0 \quad$ if $\quad|z| \geq 1 \quad$ and $\quad B(s, z)=0$. Hence, $\quad B(s, \mathrm{z})=\sum_{k=0}^{K} \sum_{m=0}^{N_{A}(M)} b_{m, k} s^{m} z^{-\tau_{k}} \neq 0 \quad$ for $\operatorname{Re} s \geq 0$ and $|z| \geq 1, \tau_{k} \in\left[0, \bar{\tau}_{k}\right], \bar{\tau}_{k}$ is a fi nite constant, $k=1,2, \ldots, K$. Q.E.D.

Proof of Theorem 2: We can see that the conditions (7) and (9) are equivalent to those of (10) and (11) of Lemma 2 [1-3]. Since Lemma 2 is a necessary and sufficient condition of the stability of two-variable polynomial $B(s, z)$, and due to Theorem 2 , the stability of $B(s, z)$ is sufficient for the quasipolynomial with multiple delays in (4), the conditions (10) and (11) are sufficien $t$ for the stability of quasi-polynomial with multiple delays in (4). Q.E.D.

The proof approaches of the above lemma and theorems are similar to those of [23-26], while the results of [26] are suitable to 2-D discrete systems only, and they cannot be employed to the 2-D polynomial $B(s, z)$ in (6) directly.

Theorem 2 provides a sufficient condition for the stability of the qu asi-polynomial with multiple delays in (4), from Theorem 2 we can obtain following corollary for networked systems.

Corollary 1: Networked system (1) is asymptotically stable, if

(i) $B(1, z)=\operatorname{det}\left(\mathbf{I}-\mathbf{A}_{0}-\sum_{k=1}^{K} \mathbf{A}_{k} z^{-\tau_{k}}\right) \neq 0,|z| \geq 1$

(ii) $B\left(s, \mathrm{e}^{\mathrm{j} \omega}\right)=\operatorname{det}\left(s \mathbf{I}-\mathbf{A}_{0}-\sum_{k=1}^{K} \mathbf{A}_{k} \mathrm{e}^{-\mathrm{j} \omega \tau_{k}}\right) \neq 0$ 
$\tau_{k} \in\left[0, \bar{\tau}_{k}\right], \bar{\tau}_{k}$ is a finite constant, $k=1,2, \ldots, K$.

For networked systems we need not expand the characteristic polynomials of the systems, we can directly test the stability conditions of Corollary 1 for them.

\section{SOME EXAMPLES}

In this section, we provide some examples illustrating the application of the results of the paper. These examples cannot be tested by the algorithms of [17-19], since the delays of networked systems in our examples are uncertain and of finite upper-bounds.

Example 1: This example is revised from Example 1 of [20], here we consider $\mathrm{n}=0$ [20], thus $K=\left(\frac{\pi}{2}-1\right)^{2}$, and we construct the networked system from the quasipolynomial of [20],

$$
B(s)=\left(s+e^{-s \tau}\right)^{2}+K
$$

We regard the quasipolynomial (13) to be the characteristic polynomial of the networked system with single delay and analyze its stability,

$$
\dot{\mathbf{x}}(t)=\mathbf{A}_{0} \mathbf{x}(t)+\mathbf{A}_{1} \mathbf{x}(t-\tau)
$$

where $\mathbf{A}_{0}=\left[\begin{array}{cc}0 & -1 \\ K & 0\end{array}\right], \mathbf{A}_{1}=\left[\begin{array}{cc}-1 & 0 \\ 0 & -1\end{array}\right], \tau \in[0,0.51]$.

From (14) and (6), we get the 2-D polynomial

$$
B(s, z)=\left(s+z^{-\tau}\right)^{2}+K
$$

From the condition (i) of Theorem 21 and (16) we have

$$
B(1, z)=(1+z)^{-2 \tau}+K \neq 0,|z| \geq 1
$$

Applying the condition (ii) of Theorem 2 we have $B\left(s, \mathrm{e}^{\mathrm{j} \omega}\right)=\left(s+\mathrm{e}^{-\mathrm{j} \omega \tau}\right)^{2}+K \neq 0$, for all $\omega \in[0,2 \pi]$ and $\operatorname{Re} s \geq 0$

The real part curve of the roots of $B\left(s, \mathrm{e}^{\mathrm{j} \omega}\right)$ is shown in Fig.1, there is no root with the real part to be larger than 0 for all $f=\frac{\omega}{2 \pi} \in[0,1]$ and $\tau \in[0,0.51]$. Thus, according to Theorem 2 , the networked system (15) is asymptotically stable. Let the initial states $\mathbf{x}(0)=\left[\begin{array}{l}1 \\ 1\end{array}\right]$ and $\tau=0.51$, simulating the networked system (15), we obtain the system states shown in Fig.2, which also verifies the system is stable.

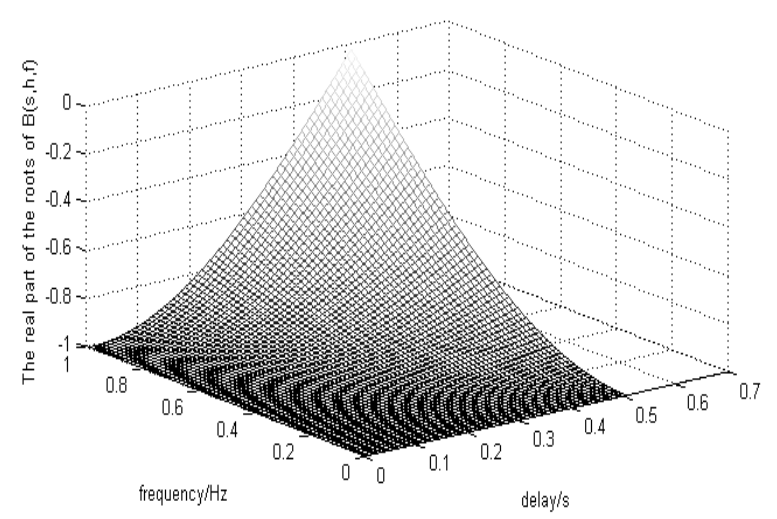

Fig.1 The real part of the roots of $B\left(s, \mathrm{e}^{\mathrm{j} \omega}\right)$ in (18) for $\tau \in[0,0.51]$

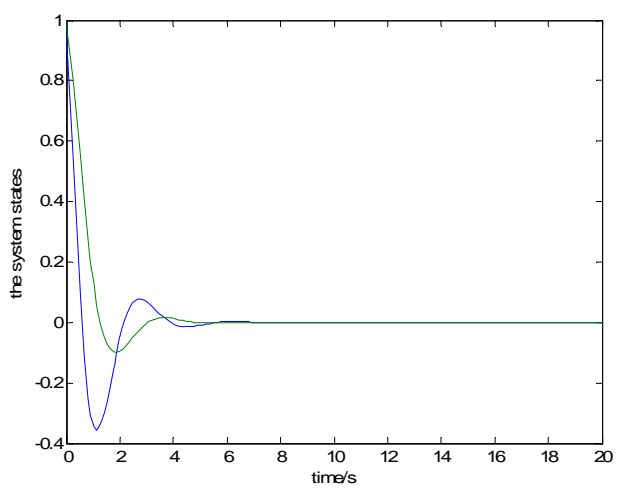

Fig.2 The system states of the networked system (15)

Example 2: Consider the networked system with two interval delays

$$
\begin{gathered}
\dot{\mathbf{x}}(t)=\mathbf{A}_{0} \mathbf{x}(t)+\mathbf{A}_{1} \mathbf{x}\left(t-\tau_{1}\right)+\mathbf{A}_{2} \mathbf{x}\left(t-\tau_{2}\right) \\
\text { where } \mathbf{A}_{0}=\left[\begin{array}{cc}
-2 & 0 \\
0 & -0.9
\end{array}\right], \mathbf{A}_{1}=\left[\begin{array}{cc}
-0.4 & 0 \\
-0.6 & -0.3
\end{array}\right],
\end{gathered}
$$

$\mathbf{A}_{2}=\left[\begin{array}{cc}-0.6 & 0 \\ -0.5 & -0.6\end{array}\right], \tau_{1} \in[0,8.9]$ and $\tau_{2} \in[0,12.2]$.

The characteristic polynomial of the system (19) is obtained as

$$
B(s)=\operatorname{det}\left(s \mathbf{I}-\mathbf{A}_{0}-\mathbf{A}_{1} \mathrm{e}^{-s \tau_{1}}-\mathbf{A}_{2} \mathrm{e}^{-s \tau_{2}}\right)
$$

From (27) and (13), we get the 2-D polynomial

$$
B(s, z)=\operatorname{det}\left(s \mathbf{I}-\mathbf{A}_{0}-\mathbf{A}_{1} z^{-\tau_{1}}-\mathbf{A}_{2} z^{-\tau_{2}}\right)
$$

From the condition (i) of Corollary 1 and (30) we have

$$
B(1, z)=\operatorname{det}\left(\mathbf{I}-\mathbf{A}_{0}-\mathbf{A}_{1} z^{-\tau_{1}}-\mathbf{A}_{2} z^{-\tau_{2}}\right) \neq 0,|z| \geq 1
$$

From the condition (ii) of Corollary 1 we have

$$
\begin{aligned}
& B\left(s, \mathrm{e}^{\mathrm{j} \omega}\right)=\operatorname{det}\left(s \mathbf{I}-\mathbf{A}_{0}-\mathbf{A}_{1} \mathrm{e}^{-\mathrm{j} \omega \tau_{1}}-\mathbf{A}_{2} \mathrm{e}^{-\mathrm{j} \omega \tau_{2}}\right) \neq 0 \\
& \text { for } \text { all } \omega \in[0,2 \pi], \quad \operatorname{Re} s \geq 0, \quad \tau_{1} \in[0,8.9] \quad \text { and } \\
& \tau_{2} \in[0,12.2] .
\end{aligned}
$$


The real part c urve of the roots of $B\left(s, \mathrm{e}^{\mathrm{j} \omega}\right)$ for $\tau_{1} \in[0,8.9]$ and $\tau_{2} \in[0,12.2]$ is shown in Fig.3, there is no root with the real part of the roots to be larger than 0 for all $f=\frac{\omega}{2 \pi} \in[0,1], \tau_{1} \in[0,8.9]$ and $\tau_{2} \in[0,12.2]$. Thus, the system (19) is asymptotically stable. In Fig.3, to show the real parts of the roots $S_{0}\left(\mathrm{e}^{\mathrm{j} \omega}, \tau_{1}, \tau_{2}\right)$ depend on the delays $\tau_{1}$ and $\tau_{2}$, we select the real parts of $s_{0}\left(\mathrm{e}^{\mathrm{j} \omega}, \tau_{1}, \tau_{2}\right)$ to be $\max _{\omega \in[0,2 \pi]} \operatorname{Re}\left[s_{0}\left(\mathrm{e}^{\mathrm{j} \omega}, \tau_{1}, \tau_{2}\right)\right]$.

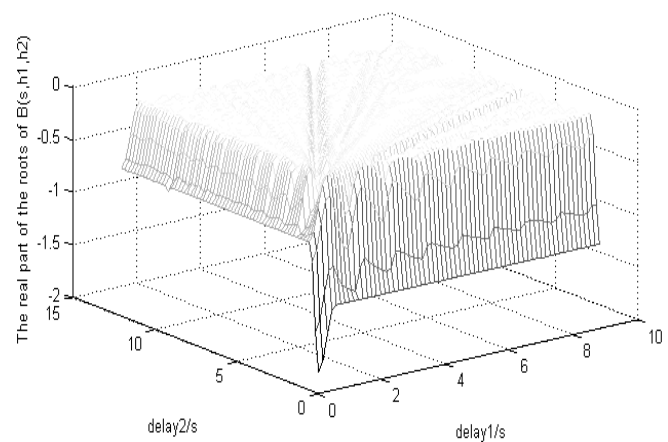

Fig.3 The real part of the roots of $B\left(s, \mathrm{e}^{\mathrm{j} \omega}\right)$ in (23) for $\tau_{1} \in[0,8.9]$ and $\tau_{2} \in[0,12.2]$

Let the initial states $\mathbf{x}(0)=\left[\begin{array}{l}1 \\ 1\end{array}\right], \quad \tau_{1}=8.9$ and $\tau_{2}=12.2$, simulating the networked system (19), we obtain the system states shown in $\mathrm{Fi}$ g.4, which also verifies the system is stable.

From Example 2 we can see that though the networked system (19) has two delays, the complexity of stability test is not very high, we have no computation complexity problem for the stability test of $t$ he networked system with multiple delays. The proposed 2-D Hurwitz-Schur stability test of 2-D hybrid polynomial $B(s, z)$ provides a n ew approach to determine the stability of the networked system with multiple delays.

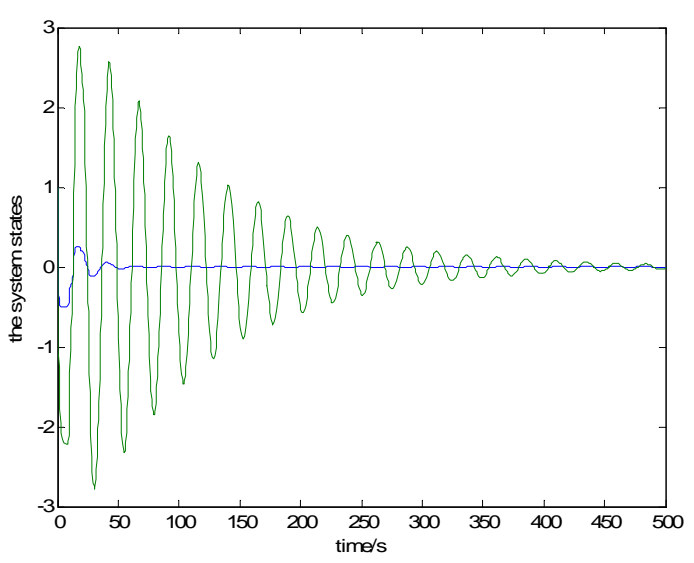

Fig.4 The system states of the networked system (19)

\section{CONCLUSIONS}

The stability conditions of networked systems with multiple delays derived from 2-D hybrid polynomial $B(s, z)$ are sufficient, we only consider the delays of the systems from zero to finite upper bound. This paper establishes the stability relationship between the characteristic polynomials of networked systems and 2-D s-z polynomials. Theorem 2 and Corollary 1 are based on the stability of 2-D s-z polynomials. The proofs and applications of the test th eorems for the networked systems with multiple delays are provided.

\section{REFERENCES}

[1] Y. Xiao, "2-D algebr aic test for robust stability of time-delay systems with interval parameters", Journal of S ystems Engineering and Electronics, Vol.17, No.4, pp.802-810, 2006.

[2] Y. Xiao, "2-D algebr aic test for robust stability of quasipolynomials with in terval parameters", Asian Journal of Control, Vol.8, No.2, pp.174-179, 2006.

[3] Y. Xiao, "Hurwitz-Schur Stability Test of Interval Bivariate Polynomials", Proceedings of IEEE International Symposium on Circuits and Systems (ISCAS2001), 2001, pp.II-829-832.

[4] V. L. Kharitonov and A. P. Zhabko, "Robust Stability of TimeDelay Systems", IEEE Trans. Automatic Control, Vol.39, No.12, pp.2388-2397, 1994.

[5] V. B. Kolmanovskii, S. Nicule scu, K. Gu, "Delay Effects on Stability: A Survey", Proc. of 38th Conference on Decision and Control, Phoenix, Arizona, USA, 1999, pp. 1993-1998.

[6] Y. Sun, J. Hsieh, and H. Yang, "On the Stability of Unc ertain Systems with Multiple Time-Varying Delays", IEEE Trans. Automatic Control, Vol.42, No.1,1997, pp.101-105.

[7] J. K. Hale and S. M. V. Lunel, Introduction to Functional Differential Equations. New York: Springer-Verlag, 1993.

[8] E. Fridman an d U. Shaked, "New bounded real lemma representations for time-delay systems and their applications," IEEE Trans. Automatic Contr., vol. 46, pp. 1973-1979, Dec. 2001.

[9] S. Boyd, L. El Ghaoui, and E. Feron et al., Linear Matrix Inequalities in System and Control Theory. Philadelphia, PA: SIAM, 1994. 
[10] S. I. Niculescu, C. E. de Souza, J. M. Dion, and L. Dugard, "Robust stability and stabilization of un certain linear systems with state delay: single delay case (I)," in Proc.IFAC Symp. Rob ust Control Design, Riode Jane iro, Brazil, Sept. 1994, pp. 469474.

[11] J. H. Su, "Further results on the robust stability of linear systems with a singl e time delay," System Control Le tt., vol. 23 , pp. 375-379, 1994.

[12] X. Li and C. E. de Souza, " Criteria for rob ust stability of uncertain linear systems with tim e-varying state delays," in IFAC 13thWorld Congr., vol. 1, San Francisco, CA, 1996, pp . $137-142$.

[13] J. H. Kim, "Delay and its time-derivative dependent robust stability of time-delayed linear systems with uncertainty," IEEE Trans. Automatic Control, vol. 46, No. 5, pp. 789-792, 2001.

[14] D. Yue and S. Won, "An improvement on 'Delay and its timederivative dependent robust stabil ity of time-delayed linear systems with uncertainty'," IEEE Trans. Automatic Control ., vol. 47, No. 2, pp. 407-408, 2002.

[15] J. Zhang, P. Tsiotras, and C. Knopse, "Stability analysis of LPV time delayed systems," Int. J .Control, vol. 75, pp. 538-558, 2002.

[16] V. Kolmanovskii, S.-I. Niculescu, and J. P. R ichard, "On the Lyapunov-Krasovskii functions for stability analysis of linear delay systems,” Int. J . Control, vol. 72, pp. 374-384, 1999.

[17] E .W. Kamen, "On the rel ationship between zero crit eria for two-variable polynomials and as ymptotic stability of delay differential equations", IEEE Trans. Automatic Control, vol.25, no.5, pp.983-984. 1980.

[18] J. K. Hale, E. F. Infante, and F. S. P. Tsen, "Stability in linear delay equations,” J. Math. Anal. Appl., vol. 105 , pp. 533-555, 1985.

[19] J. Chen, S. I. Niculescu and P. L. Fu, "Robust Stability of QuasiPolynomials: Frequency-Sweeping Conditions and Vert ex Tests", IEEE Transactions on Automatic Control, Vol. 53, No. 5, pp.1219- 1234, 2008.

[20] M. Fu, A.W. Olbrot, M. P. Polis, "Robust stability for timedelay systems: the edg e theorem and gr aphical tests", IEEE Trans. Automatic Control, Vol.34, No.8, pp.613-82 0, 1989.

[21] J. A. Marshall, M. E. Broucke, and B. A. Fran cis, "Formations of vehicles in cyclic pursuit," IEEE Transactions on Automatic Control, vol. 49, pp. 1963-1974, 2004.

[22] J. D. Birdwell, J. Chiasson, Z. Tang, C. Abdallah, M. M. Hay at, and T. Wang, "Dynamic time delay models for load balancing. parti: Deterministic models," in Advances in Time-Delay Systems, S.-I.Niculescu and K. Gu, Eds. Berlin: Springer, 2004, pp. 355-370.

[23] Y. Xiao, R. Unbehauen and X. Y. Du, "Robust Hurwitz stability of conditions of polytopes of bivariate poly nomials", Proceedings of 38th IEEE Conference on Decision and Control, Phoenix, Arizona, 1999, pp. 5034-5035.

[24] Y. Xiao, R. Un behauen, X. Du, "Robust Hurwitz Stab ility of Conditions of Polytopes of Bivariate Polynomials", Proceedings of 38th I EEE Conference on Decision and Control, 1999, pp.5034-5035.

[25] Y. Xiao and R. Unbehauen, "New Stability Test Algorithm for Two-Dimensional Digital Filters", IEEE Trans. Circuits and Systems I, Vol.45, No.7, pp.739-740, 1998.
[26] D. L. A. Davis, "Correct proof of Huang's theorem on stability", IEEE Trans. on Acoustics, Speech, and Signal Processing, vol.24, no.10, pp.425-426, 1976.

\section{Creative Commons Attribution License 4.0 (Attribution 4.0 International, CC BY 4.0)}

This article is published under the terms of the Creative Commons Attribution License 4.0 https://creativecommons.org/licenses/by/4.0/deed.en US 